\title{
Emerging Technologies for Monitoring Behavioural and Psychological Symptoms of Dementia
}

\author{
Tarik Qassem \\ Warwick Medical School \\ University of Warwick \\ Coventry, UK \\ T.Qassem@warwick.ac.uk \\ Philip Moore \\ School of Information Science and Engineering \\ Lanzhou University \\ Lanzhou, China \\ drpmlzu@yahoo.co.uk
}

\author{
George Tadros \\ Warwick Medical School \\ The University of Warwick \\ Coventry, UK \\ E-mail: george.tadros@nhs.net \\ Fatos Xhafa \\ Technical University of Catalonia \\ C. Jordi Girona, 08034 \\ Barcelona, Spain. \\ fatos@1si.upc.edu
}

\begin{abstract}
Behavioural and psychological symptoms of dementia (BPSD) are complex array of symptoms that have devastating impact on patients, carers and their loved ones. In this paper we argue that with the combined use of pervasive computing and big data, we could make significant progress in the diagnosis of the causes of BPSD, monitoring response to treatment and helping in the prevention of these symptoms. We review the available technologies, such as Cloud computing and context aware systems, and how they could help in managing and hopefully preventing the Behavioural and Psychological Symptoms of Dementia.
\end{abstract}

Keywords-Dementia, Alzheimer's disease, Neuropsychiatric symptoms, BPSD, Monitoring Behavioural and Psychological Symptoms of Dementia, pervasive computing, wearable computing, ubiquitous computing, data mining, machine learning, big data)

\section{INTRODUCTION}

Behavioural and psychological symptoms of dementia (BPSD) are a heterogeneous group of clinical phenomena that is characterised by disturbed emotions, mood, perception, thought, motor activity, diurnal rhythm and altered personality traits [1], [2]. BPSD are a source of significant distress and poor quality of life (QoL) to both individuals with dementia as well as their carers [2], [3]. They are associated with increased mortality rate and rapid deterioration of cognition [2], [4], [5]. Moreover, they are major reason for early institutionalisation of patients [6], which in turn significantly increases the overall cost of dementia [7].

BPSD are very common regardless of the type or the stage of dementia. Even in the early stages of cognitive impairment, BPSD are frequent with estimated rates of $35-85 \%$ in individual with mild cognitive impairment. While in community-dwelling patients, BPSD are generally more frequent (56-98\%), it is almost universal in patients who are in hospital or live in a long-term care facility (91-96\%) [8]. BPSD requires continuous care of patients of dementia causing a significant burden to all who are involved. With the fast development and maturity of Internet-based technolgies, there are emerging trends of using such technological advances for supporting doctors, nurses, carers in their everyday tasks with patients of dementia. While such emerging technlogical solutions cannot provide automated solutions, they can be helpful in a number of ways to alleviate the burden of premature institutionalisation of patients of dementia as well as incrementing the QoL to patients themselves and their carers.

In this paper we analyse the main requirements deriving from BPSD and their transalation into functional requirements of computational solutions. Indeed, we argue that the combination of pervasive computing, big data processing and machine learning, we could make significant progress in the early diagnosis of the causes of BPSD, monitoring response to treatment and helping in the prevention of these symptoms. Besides

The rest of the paper is organized as follows. In Sect. II we analyse the causes of BPSD grouped into four main areas. Ambulatory monitoring and telemetry are discussed in Sec III. Then, in Sect. IV we discuss how monitoring and telemetry can be handled through Big Data approaches where emerging applications are discussed as well. We end the paper in Sect. VII with some conclusions and outlook for future work.

\section{CAUSES OF BPSD}

The causes of BPSD fall under four main areas [9]. These areas are physical disease, psychological/psychiatric disorders, environmental causes and dysfunctional communication.

\section{A. Physical problems}

Acute physical issues like pain, urinary tract infection, constipation and medications/drugs can affect behaviour whether directly or indirectly through causing delirium. Individuals with dementia have a five times higher risk of developing delirium compared to normal old people [10]. That delirium usually resolves when the underlying acute physical 
cause is treated. In severe dementia, delirium can be triggered by quite minor physical causes leading to possible challenging behaviour. Unfortunately, at least $50 \%$ of patients with dementia in institutions who exhibit BPSD suffer from undiagnosed physical condition that could contribute to the development of their BPSD [11], [12]. In community-residing older adults with dementia the rate of undiagnosed relevant medical condition is $36 \%$ [13].

\section{B. Psychological / psychiatric conditions}

The commonest psychiatric conditions associated with dementia are anxiety, depression and psychosis.

Depression is more likely to occur in dementia than in healthy elderly. This manifests itself as aggression, anxiety, irritability, crying, calling for help, withdrawal, anorexia, poor sleep, reduced conversation, non-compliance or clinging to people for reassurance.

Anxiety is more likely to develop in people with premorbid anxious personalities. Patients with anxiety tend to startle easily, worry, seek reassurance, are fearful, restless, and hypervigilant. It is worth noted that anxiety can be a symptom of depression in elderly and can be the most manifest sign.

Psychosis usually manifest with two groups of symptoms, delusions or hallucinations. Delusions are usually persecutory. Hallucinations are often of people or animals that are usually threating or frightening but can also be pleasant.

Even in severe cases of dementia, patients retain some selfawareness, which can be a cause of psychological distress, for example embarrassment over incontinence or the need of personal assistance may lead to aggression. Other causes for challenging behaviour in dementia are feeling of loneliness and boredom.

\section{Environmental causes}

Dementia sufferers are quite sensitive to environmental changes. The mere admission in a hospital or care-home can precipitate challenging behaviour. It can be a frightening experience especially when they don't know the reason for admission or the people around them.

When care-homes are under-lit or have no signage, patients seem to lose their way and wander with incidences of accidental urination or defecation in inappropriate places. Other challenging behaviour can emerge when helpers try to approach them.

Dementia sufferers need their own personal space and an environment where they can explore. Most of them show fewer problems when left to their own devices. Restrictive environment where people get in each other's way can raise conflicts and cause challenging behaviour. They may think that others have come to inhabit their home and so try to eject them. Other environmental conditions to consider are room temperature whether too hot or cold and constant noise that may cause distress and annoyance.

\section{Communication difficulties}

Failing to express oneself through language is tremendously frustrating and the patient is only left with non- verbal communication as a way of interaction. This can result in depression, which could manifest as challenging behaviour.

\section{AMBULATORY MONITORING AND TELEMETRY}

Ambulatory monitoring of behaviour in dementia depends on telemetrics, which is automatic measurement and transmission of data from remote sources [14]. These systems use portable, wearable and ubiquitous computed devices, allowing behavioural scientists to monitor behavioural, cognitive, affective and psychological responses of individuals in various situations [15], [16].

In 2006, Nusser et al. made the assertion that with the technology available at that time it was not uncommon to create a comfortable device that records and transmits a stream of video data of what the person sees, audio data of what is being heard and said, accelerometer data of muscle activities, physiological data such as heart rate, GPS information on the subject's location and the feelings of the subject reported to a mobile computer device user interface. With such technology, all of the information collected can be chronologically stamped and synchronised so their relation to time and surrounding is recognised and confirmed [16], [17] (in computational terms this is referred to as contextual information).

Like other tools used in psychometrics, telemetrics needs to be valid, reliable, and should not affect the behaviour that is measured. These are also referred to as ecological validity, repeated measurement, and reactivity to measurement. There is another challenge to telemtry applied to patients of dementia, namely, most of the of patients of dementia do not collaborate in telemetrics and therefore the technology should be ideally fully non-intrusive, so it does not affect the patient's behaviour.

Ecological validity means that the methods and the setting of the study must approximate the real-world that is being examined [18], which would mean that the device makes observations in the real world where behaviour, and all of its structural and functional relationships, occur naturally.

One of the challenges that is associated with repeated measurement is the inherent variability of individual's behaviours, as it tends to vary in response to situations and across time [19]. While the nomothetic approach of behavioural assessment relies on population averages captured at one or at a few time points, using a limited number of dependent variables [20], the idiographic approach aims to capture the uniqueness of individual behaviour through sampling behaviour that focuses on the prospective, multivariate, time-dependent variation within individuals [21].

Reactivity to measurement refers to the effect of the act of measurement on the behaviour measured [22]. Behavioural psychologists have long advocated unobtrusive observation procedures to avoid reactivity to measurement [16].

In their review, Goodwin et al. [16] how pervasive computing could be used for monitoring of behaviour. They described two complementary approached; wearable computing and ubiquitous computing [16]. 


\section{A. Ubiquitous computing}

Ubiquitous computing means that computers "weave themselves into the fabric of everyday life until they are indistinguishable from it" [23]. Over the past decade, researchers have been working on integrating ubiquitous sensing technologies in surrounding environment to create monitor rooms and smart laboratories where environmental and behavioural monitoring can be done wirelessly and passively [24]-[26]. A single synchronised set of information can be obtained when all of these sensors continuously and automatically send out their recorded data to a nearby computer network [16].

\section{B. Pervasive computing}

For pervasive computing systems to be able to capture data, they would rely on different types of sensors. That range from infrared thermometers, body fat analysers, radio-frequency identification, accelerometers, gyroscopes, and passive infrared sensors. These sensors are either wearable sensors or sensors distributed across the environment. The wearable sensors are usually used for capturing physiological measurements such as heart rate, electricity skin conduction, respiratory rate, or even electrical signals from the surface of the head (EEG).

Environment-embedded sensors are usually used for capturing or measuring physical phenomena such as motion, degree of noise or intensity of light. Wearable computing are perception systems that can be embedded into wearable items like jewellery, gloves, clothes or shoes [27]. For example, small unnoticeable on-body wireless sensors have been developed to record respiratory, cardiovascular and muscle activity as well as skin conductivity of freely mobile people [28]. Tiny accelerometers and actigraphs monitoring and quantifying physical activities have been embedded into wearable bracelets, wristbands and belts. They can objectively measure dynamic activities (including climbing stairs and walking) and body posturing [29]. Unobtrusive recording microphones have also been integrated into wearable computing platforms where the client's voice and remarks are recorded together with ambient audio events in the surrounding environment [30]. Miniature sensitive infra-red cameras are embedded into eye-glasses to detect where a user is looking and what they are looking at [31], [32].

There is now a growing market for consumer wearable sensors which range from wristbands that are able to measure heart rate, calories burned, depth of sleep to newly developed user interface devices that are able to remotely control other computer devices through brain electrical activity. There is merging evidence that commercial EEG headsets are able indeed to detect and differentiate emotions such as excitement or frustration in nonclinical population [33].

These sensors could detect wide range of behaviours that are associated with BPSD. For more details, the reader is referred to Table 1.

\section{BIG DATA}

Nowadays, behavioural scientists and clinicians can observe human behaviour on a scale that has never been achieved before and in the finest temporal granularity. This is already happening as we could observe behavioural trends in social media. This gives us the opportunity of assessing the course of treatment and disease in a temporal continuum, instead of relying on glimpses [35]. With the combined force of telemetry and Big Data the care for those who suffer from dementia could be revolutionised.

Big Data refers to the process of extraction, collection, cleaning and transforming, storage, management, analyses, indexing and searching, as well as visualisation of complex data.

Data mining and machine learning are the two main applications for Big Data. Data Mining paradigm is pattern recognition through use of large datasets to recognise unnoticed patterns between different variables in the data. Meanwhile Machine Learning paradigm is to "learn" new properties about known entities. Both Data Mining and Machine Learning are heavily used in marketing and banking sectors for recognising shopping behaviour and for making credit decisions. In fact, the emergence of Cloud computing systems enables not only to process and discover knowledge from the data from a historical perspective, say within the last week, such as patterns of patient's behaviour, but it is also possible to process the data streams and spot events that indicate abrupt changes in the patient behaviour so that medical teams can react accordingly.

The fusion of multimodal sensor network and the analytic power of data mining and machine learning give us a potentially very effective way in improving the quality of life of individuals who suffer from dementia by delaying as much as possible their institutionalisation in hospitals or care centers. Yet, much research is needed to improve the adoption of these methods to the needs of patients with dementia. Indeed, unlike monitoring of other phenomena, patient monitoring requires a threshold of accuracy, detecting missing data, potential errors with the data as well as data lineage and provenance to ensure the availability and readiness of data at long run. Also, the pervasive nature of these technologies requires a rigorous debate on different ethical aspects to their use.

TABLE I. POSSIBLE SENSORS TO CAPTURE BPSD (SOURCE: [34])

\section{Sensor Behaviour}

\begin{tabular}{ll} 
Motion & \multicolumn{1}{l}{ Restlessness } \\
Radar & Tapping/Banging \\
GPS tracking & Wandering \\
Acoustic & Vocalisation \\
Pressure (bed) & Sleep disturbance \\
Video & Daily activities
\end{tabular}

As an illustrative example, let us consider wandering. Wandering [36] can be modeled and computed to a satisfactory level of accuracy. The objective here is to identify 
patterns of wandering from which states of the patient of dementia can be inferred, such as agitation. In order to model and deduce the patterns of wandering, it is necessary to make tracking of the patient and get the temporary points about his positions. There are different ways of doing this, for instance through GPS tracking or video monitoring [37]. Such computational models are nowadays feasible due the unprecedented increase on both storage and computing capacity of todays ICT infrastructures based on Cloud computing systems.

\section{A. Assessment and management of BPSD}

In clinical practice, assessment of BPSD requires a thorough clinical history taking, examining patient's subjective experience, and objective behaviour. Collateral history from a reliable family member or carer is essential to obtain adequate description of behavioural disturbances and their context. Whenever possible, patients should be encouraged to express their own concerns. That could give the clinician valuable insight to the patients inner experience. In addition to that clinician usually rely on Functional Behaviour Assessment, also known as ABC approach, which is an acronym for Antecedent, Behaviour and Consequence.

Functional Behaviour Assessment requires clear specification of a problem behaviour ('B') that is understood in terms of the observed influence of events that precedes it (antecedents 'A'), and the events consequent ('C') upon it [36]. Traditional 'ABC' Functional Behavioural Assessment underlying hypothesis is that behaviour is always observable and follows a linear pattern. However, this is not necessarily true for the development and maintenance of challenging behaviour in dementia [37]. Furthermore, a particular behaviour may have different functions for different individuals, or more than one function for a particular people for different occasions [38]. That makes the Functional Behaviour Assessment complex task.

Relying on objective assessment of behaviour could be the best method to assess the severity, frequency and the nature of the challenging behaviour. With objective assessments, it is more possible to elicit the end precedents and a consequence of that challenging behaviour. However, continuous observation of challenging behaviour could be arduous in itself due to the high resource costs. That could be the case if that behaviour is not frequent or follow a pattern that's not easily recognised.

\section{B. Behavioural and Psychological Symptoms of Dementia and the use of big Data}

With the use of pervasive computing techniques such as wearable and ubiquitous computing, the data generated could be analysed in a large scale, and to extract meaningful indices[35], such as an "agitation index", or a "distress index", rather than depending on the report from carers. Clinicians can then track patients' symptoms in fine-granular temporal resolution. They can explore interdependencies between different factors that could affect those symptoms, such as social activities, medications, and psychical conditions. Machine learning techniques could be able to alert early warning signs, so they could act proactively. In this case, they can intervene earlier. At the same time, regular appointments can be spaced further apart, which would be significantly cheaper for the health economy[35].

The use of data generated by pervasive computing gives a continuous temporal, but contemporary picture to patients condition and what factors could be influencing any change. Mining patients' individual data available as electronic health records, enables the system to recognise any anomaly in individuals' behaviours and possible risk factors, such as recent start of medication or recent infection. While, with machine learning, an intelligent system could classify patients according to risk score and predict patients' individual risk, for example falls or having an underlying infection as a cause of the BPSD. That needs integration of data from different source including patients' health record as well as the ability to recognise patterns in the records of patients of the same characteristics.

\section{EMERGING APPLICATIONS}

There have been few applications in attempt to use ambulatory monitoring in the assessment of challenging behaviour. Of the symptoms of BPSD, wandering and agitation are the ones most studied. But for the propose of this paper we will focus on agitated behaviour.

The literature[39] [40][41] on monitoring of agitation and aggression in patients with dementia has focused on the use of Scale to assess Observed Agitation in Persons with Dementia of the Alzheimer Type (SOAPD)[42]. The SOAPD rates the following aspects of agitated behaviours:

- Total Body Movements

- Up and Down Movements

- Repetitive Motions in Place

- Outward Motions

- High Pitched/Loud Words

- Repetitive Vocalisation

- Negative Words.

This scale was adapted for the purpose of automatic monitoring of agitation in patients with dementia by Fook et al [39]. They also explored the use of the video recognition in assessment of agitated behaviour in patients with dementia. They reviewed the ontology of agitation in dementia, its determinants, its digital representation and how the pattern recognition system could identify early precursor. Their work lays the theoretical foundation for future use of technology in assessment of agitated behaviour in dementia [39].

Another described method used for assessing challenging behaviour is wearable computing. Plötz et al [43] described a system they developed to recognise challenging behaviour in this in children with developmental disorder. They examined three types of challenging behaviour: aggression, disruption, and self-injurious behaviour. They had three phases of the experiments. Phase 1 aimed to training the computer system to detect simulation of the challenging behaviour. They instructed experienced staff member to simulate the three types of behaviour. In this phase, machine learning could automatically detect the simulated challenging behaviour in $95 \%$ of time. It 
was also able to differentiate between the three types of behaviours with the precision of approximately $80 \%$. In the second phase of the experiments the researchers instructed the participants just to simulate taking part in normal daily routine activities.

The aim of the second phase was to examine the rate of false positive detection in challenging behaviour. The system achieved negligible false positive results. In the third phase of the experiments the researchers tested the developed system on real patients with challenging behaviour and they achieved approximately $70 \%$ precision rate. The authors explanation of the lower precision rates in the third phase was failure of one of the subjects to tolerate an ankle sensor, hence lowering the detection rate and real patients.

\section{A. Possible applications for current technology}

Even the currently available sensors and computing technologies (see Table 2 in the appendix) could hold the key for the future development in managing one of the difficult aspects of dementia, such as BPSD.

\section{B. Contextual Information}

Potential future applications will incorporate the ability to utilise an ever increasing range of dta (or contextual information). Location is central to context; context however includes more than just location; in actuality, almost any information available at the time of an interaction can be viewed as contextual information including:

- The variable tasks demanded by users

- The diverse range of mobile devices and the associated service infrastructure(s) in LSDS incorporating Wide Area Networks (WAN), Local Area Networks (LAN), and personal Area Networks (PAN) implemented in internet and Internet systems

- Resource availability (connectivity, battery condition, display, network, and bandwidth etc)

- Nearby resources (accessible devices and hosts including $\mathrm{I} / \mathrm{O}$ devices

- The physical situation (temperature, air quality, light, and noise level etc)

- The social situation (who you are with, people nearby etc - proximate information)

- Spatial information (location, orientation, speed and acceleration etc)

- Temporal information (time of the day, date, and season of the year)

- Physiological measurements (blood pressure, heart function - electrocardiography (ECG or EKG from the German Elektrokardiogramm ), cognitive functions related to brain activity (EEG from Electroencephalography ), respiration, galvanic skin response, and motor functions including muscle activity)
- Cognitive and abstract contextual information such as an individuals emotional responses, intuition, feelings, and sensibilities expressed in terms of semantic terminologies

As can be seen from the extensive range of potential data emerging and future applications hold the promis of greatly enhanced monitoring ability and the capacity to realise increased computational intelligence [52].

\section{CONCLUSION}

Pervasive computing is infiltrating our lives day by day, as it's becoming more accessible and cheaper. Although there has been growing interest in wearable computers and devices that could aid in maintaining our health and fitness, yet, there has been limited research in the use of pervasive and wearable computing in the dementia care.

We believe there are huge opportunities for these technologies to be exploited in dementia care, especially in diagnosis, management and prevention of BPSD. However, for pervasive computing to work, it will need the use of mining big data sets in order to have a more efficient Machine learning algorithms. Additionally, accuracy, non-obtrusiveness, privacy are issues to be considered in such computational BPSD systems.

In our future work we would like to establish a rigorous computational model comprising BPSD requirements and its translation to a software system that can support medical teams of dementia.

\section{REFERENCES}

[1] S. I. Finkel, J. Costa e Silva, G. Cohen, S. Miller, and N. Sartorius, "Behavioral and psychological signs and symptoms of dementia: a consensus statement on current knowledge and implications for research and treatment," Int. Psychogeriatrics, vol. 8, no. S3, pp. 497-500, 1997.

[2] J. Cerejeira, L. Lagarto, and E. B. Mukaetova-Ladinska, "Behavioral and psychological symptoms of dementia," Front. Neurol., vol. 3, 2012.

[3] S.-H. Ryu, J. H. Ha, D.-H. Park, J. Yu, and G. Livingston, "Persistence of neuropsychiatric symptoms over six months in mild cognitive impairment in community-dwelling Korean elderly," Int. Psychogeriatrics, vol. 23, no. 02, pp. 214-220, 2011.

[4] T. C. Russ, G. D. Batty, and J. M. Starr, "Cognitive and behavioural predictors of survival in Alzheimer disease: results from a sample of treated patients in a tertiary - referral memory clinic," Int. J. Geriatr. Psychiatry, vol. 27, no. 8, pp. 844-853, 2012.

[5] E. A. Weamer, J. E. Emanuel, D. Varon, S. Miyahara, P. A. Wilkosz, O. L. Lopez, S. T. DeKosky, and R. A. Sweet, "The relationship of excess cognitive impairment in MCI and early Alzheimer's disease to the subsequent emergence of psychosis," Int. psychogeriatrics, vol. 21, no. 01, pp. 78-85, 2009.

[6] D.-C. Chan, J. D. Kasper, B. S. Black, and P. V Rabins, "Presence of behavioral and psychological symptoms predicts nursing home placement in community-dwelling elders with cognitive impairment in univariate but not multivariate analysis," Journals Gerontol. Ser. A Biol. Sci. Med. Sci., vol. 58, no. 6, pp. M548-M554, 2003.

[7] N. Herrmann, K. L. Lanctôt, R. Sambrook, N. Lesnikova, R. Hébert, P. McCracken, A. Robillard, and E. Nguyen, "The contribution of neuropsychiatric symptoms to the cost of dementia care,” Int. J. Geriatr. Psychiatry, vol. 21, no. 10, pp. 972-976, 2006.

[8] G. Frisoni, L. Rozzini, A. Gozzetti, G. Binetti, O. Zanetti, A. Bianchetti, M. Trabucchi, and J. Cummings, "Behavioral 
syndromes in Alzheimer's disease: description and correlates," Dement. Geriatr. Cogn. Disord., vol. 10, no. 2, pp. 130-138, 1999.

[9] A. Krishnamoorthy and D. Anderson, "Managing challenging behaviour in older adults with dementia," Prog. Neurol. Psychiatry, vol. 15, pp. 20-26, 2011.

[10] M. G. Cole, "Delirium in elderly patients," Am. J. Geriatr. psychiatry, vol. 12, no. 1, pp. 7-21, 2004.

[11] E. B. Larson, B. V Reifler, S. M. Sumi, C. G. Canfield, and N. M. Chinn, "Diagnostic evaluation of 200 elderly outpatients with suspected dementia," J. Gerontol., vol. 40, no. 5, pp. 536-543, 1985.

[12] P. M. Doraiswamy, J. Leon, J. L. Cummings, D. Marin, and P. J. Neumann, "Prevalence and impact of medical comorbidity in Alzheimer's disease," Journals Gerontol. Ser. A Biol. Sci. Med. Sci., vol. 57, no. 3, pp. M173-M177, 2002.

[13] N. Hodgson, L. N. Gitlin, L. Winter, and K. Czekanski, "Undiagnosed illness and neuropsychiatric behaviors in communityresiding older adults with dementia," Alzheimer Dis. Assoc. Disord., vol. 25 , no. 2 , p. 109,2011

[14] Princeton University, "WordNet Search - 3.1," 2014. [Online].

http://wordnetweb.princeton.edu/perl/webwn?s=telemetry.

[Accessed: 05-Apr-2014].

[15] S. S. Intille, "Technological innovations enabling automatic, context-sensitive ecological momentary assessment," in The science of real-time data capture: Self-reports in ..., 2007, pp. 308-337.

[16] M. S. Goodwin, W. F. Velicer, and S. S. Intille, "Telemetric monitoring in the behavior sciences.," Behav. Res. Methods, vol. 40, pp. 328-341, 2008.

[17] S. M. Nusser, S. S. Intille, and R. Maitra, "Emerging technologies and next-generation intensive longitudinal data collection," Model. intensive Longitud. data, pp. 254-277, 2006.

[18] M. B. Brewer, "Research design and issues of validity," Handb. Res. methods Soc. Personal. Psychol., pp. 3-16, 2000.

[19] P. B. Baltes, H. W. Reese, and J. R. Nesselroade, Life-span developmental psychology: Introduction to research methods. Psychology Press, 2014.

[20] J. T. Lamiell, Beyond individual and group differences: Human individuality, scientific psychology, and William Stern's critical personalism. Sage, 2003.

[21] P. C. M. Molenaar, "A manifesto on psychology as idiographic science: Bringing the person back into scientific psychology, this time forever," Measurement, vol. 2, no. 4, pp. 201$218,2004$.

[22] D. T. Campbell, J. C. Stanley, and N. L. Gage, Experimental and quasi-experimental designs for research. Houghton Mifflin Boston, 1963.

[23] M. Weiser, "The computer for the 21st century," Sci. Am., vol. 265, no. 3, pp. 94-104, 1991.

[24] G. Abowd, C. Atkeson, and A. Bobick, "Living laboratories: the future computing environments group at the Georgia Institute of Technology," ... factors Comput. ..., p. 215, 2000.

[25] S. S. Intille, K. Larson, J. S. Beaudin, J. Nawyn, E. M. Tapia, and P. Kaushik, "A living laboratory for the design and evaluation of ubiquitous computing technologies," in CHI'05 extended abstracts on Human factors in computing systems, 2005, pp. 1941-1944.

[26] A. P. Pentland, "Smart rooms," Sci. Am., vol. 274, no. 4, pp. 54-62, 1996.

[27] F. H. Wilhelm, W. T. Roth, and M. A. Sackner, "The LifeShirt An Advanced System for Ambulatory Measurement of Respiratory and Cardiac Function," Behav. Modif., vol. 27, no. 5, pp. 671-691, 2003.

[28] J. Healey, "Future possibilities in electronic monitoring of physical activity.," Res. Q. Exerc. Sport, vol. 71, no. 2 Suppl, pp. S137-45, 2000
[29] L. Bao and S. S. Intille, "Activity recognition from userannotated acceleration data," in Pervasive computing, Springer, 2004, pp. 1-17.

[30] M. R. Mehl, J. W. Pennebaker, D. M. Crow, J. Dabbs, and J. H. Price, "The Electronically Activated Recorder (EAR): A device for sampling naturalistic daily activities and conversations," Behav. Res. Methods, Instruments, Comput., vol. 33, no. 4, pp. 517-523, 2001.

[31] C. Dickie, R. Vertegaal, J. S. Shell, C. Sohn, D. Cheng, and O. Aoudeh, "Eye contact sensing glasses for attention-sensitive wearable video blogging," in CHI'04 extended abstracts on Human factors in computing systems, 2004, pp. 769-770.

[32] R. Vertegaal, R. Slagter, G. Van der Veer, and A. Nijholt, "Eye gaze patterns in conversations: there is more to conversational agents than meets the eyes," in Proceedings of the SIGCHI conference on Human factors in computing systems, 2001, pp. 301308.

[33] D. Cernea, A. Kerren, and A. Ebert, "Detecting insight and emotion in visualization applications with a commercial EEG headset," in SIGRAD 2011 Conference on Evaluations of Graphics and Visualization-Efficiency, Usefulness, Accessibility, Usability,(Stockholm, Sweden), 2011, pp. 53-60.

[34] M. Yefimova and D. L. Woods, "Using Sensor Technology to Monitor Disruptive Behavior of Persons with Dementia," in 2012 AAAI Fall Symposium Series, 2012.

[35] A. Markowetz, K. Błaszkiewicz, C. Montag, C. Switala, and T. Schläpfer, "Psycho-Informatics: Big Data Shaping Modern Psychometrics," Med. Hypotheses, 2014.

[36] C. K. Lai and D. G. Arthur, "Wandering behavior in people with dementia," Journal of Advanced Nursing," Vo. 44/2, 2003, pp: 173182.

[37] Intelligent Robotics and Applications Lecture Notes in Computer Science Volume 7102, 2011, pp 116-124 A Method for Wandering Trajectory Detection in Video Monitor Ruohong Huan, Zhehu Wang, Xiaomei Tang, Yun Pan

[38] G. Stokes, Challenging behaviour in dementia: a personcentred approach. Speechmark, 2000.

[39] E. Moniz-Cook, R. Woods, and E. Gardiner, "Staff factors associated with perception of behaviour as' challenging'in residential and nursing homes," Aging Ment. Health, vol. 4, no. 1, pp. 48-55, 2000 .

[40] E. Moniz - Cook, G. Stokes, and S. Agar, "Difficult behaviour and dementia in nursing homes: five cases of psychosocial intervention," Clin. Psychol. Psychother., vol. 10, no. 3, pp. 197-208, 2003.

[41] V. Foo Siang Fook, S. C. Tay, M. Jayachandran, J. Biswas, and D. Zhang, "An ontology-based context model in monitoring and handling agitation behavior for persons with dementia," in Pervasive Computing and Communications Workshops, 2006. PerCom Workshops 2006. Fourth Annual IEEE International Conference on, 2006, p. 5-pp.

[42] P. Moore, A. Thomas, G. Tadros, F. Xhafa, and L. Barolli, "Detection of the onset of agitation in patients with dementia: realtime monitoring and the application of big-data solutions," Int. J. Space-Based Situated Comput., vol. 3, no. 3, pp. 136-154, 2013.

[43] P. Moore, F. Xhafa, L. Barolli, and A. Thomas, "Monitoring and Detection of Agitation in Dementia: Towards RealTime and Big-Data Solutions," in P2P, Parallel, Grid, Cloud and Internet Computing (3PGCIC), 2013 Eighth International Conference on, 2013, pp. 128-135.

[44] A. C. Hurley, L. Volicer, L. Camberg, J. Ashley, P. Woods, G. Odenheimer, W. L. Ooi, K. McIntyre, and E. Mahoney, "Measurement of Observed Agitation in Patients With Dementia of the Alzheimer Type," J. Ment. Health Aging, vol. 5, no. 2, pp. 117 134, 1999.

[45] T. Plötz, N. Y. Hammerla, A. Rozga, A. Reavis, N. Call, and G. D. Abowd, "Automatic assessment of problem behavior in individuals with developmental disabilities," in Proceedings of the 
2012 ACM Conference on Ubiquitous Computing, 2012, pp. 391400.

[46] P. Kapusta, L. Regier, J. Bareham, and B. Jensen, "Behaviour management in dementia," Can. Fam. Physician, vol. 57, no. 12, pp. 1420-1422, 2011.

[47] F. H. Wilhelm, M. C. Pfaltz, P. Grossman, and W. T. Roth, "Distinguishing emotional from physical activation in ambulatory psychophysiological monitoring.,"Biomed. Sci. Instrum., vol. 42, pp. 458-463, 2006.

[48] M. F. Valstar and M. Pantic, "Fully automatic recognition of the temporal phases of facial actions," Syst. Man, Cybern. Part B Cybern. IEEE Trans., vol. 42, no. 1, pp. 28-43, 2012.
[49] M. S. Bartlett, G. C. Littlewort, M. G. Frank, and K. Lee, "Automatic Decoding of Facial Movements Reveals Deceptive Pain Expressions," Curr. Biol., vol. 24, no. 7, pp. 738-743, 2014.

[50] M. F. Valstar, M. Mehu, B. Jiang, M. Pantic, and K. Scherer, "Meta-analysis of the first facial expression recognition challenge," Syst. Man, Cybern. Part B Cybern. IEEE Trans., vol. 42, no. 4, pp. 966-979, 2012

[51] L. LeResche, "Facial Behaviors Related to Pain in the Elderly1," Gerodontology, vol. 3, no. 1, pp. 83-86, 1984.

[52] . Moore, Philip. "Intelligent Context: The Realization of Decision Support Under Uncertainty." Inter-cooperative Collective Intelligence: Techniques and Applications. Springer Berlin Heidelberg, 2014. 111-139. 


\section{Appendix}

TABLE II. CAUSES OF BPSD AND POSSIBLE WAYS OF DETECTION USING SMART TECHNOLOGIES

\begin{tabular}{|c|c|}
\hline $\begin{array}{c}\text { Selected triggering factors in behavioural and } \\
\text { psychological symptoms of dementia [44] }\end{array}$ & How monitoring technology could help \\
\hline Psychosocial & \multirow{4}{*}{$\begin{array}{l}\text { Emerging evidence that ambulatory } \\
\text { monitoring could distinguish emotional } \\
\text { from physical activation }[45]\end{array}$} \\
\hline - $\quad$ Distress & \\
\hline - $\quad$ Anxiety and depression & \\
\hline - $\quad$ Paranoia & \\
\hline \multicolumn{2}{|l|}{ Environmental } \\
\hline \multicolumn{2}{|l|}{ - $\quad$ "Bad" company } \\
\hline - $\quad$ Boredom & $\begin{array}{l}\text { Quite environment detected by sensors } \\
\text { that precedes the challenging behaviour }\end{array}$ \\
\hline - $\quad$ Confusing surroundings & Detection of wandering pattern \\
\hline - $\quad$ Excessive demands & \\
\hline - $\quad$ Lack of routine & \\
\hline - Inadequate lighting & $\begin{array}{l}\text { Low lighting detected by sensors that } \\
\text { precedes the challenging behaviour }\end{array}$ \\
\hline - Loneliness & $\begin{array}{l}\text { Quiet environment detected by sensors } \\
\text { that precedes the challenging behaviour }\end{array}$ \\
\hline - $\quad$ Noise & $\begin{array}{l}\text { Excessive sounds detected by sensors that } \\
\text { precede the challenging behaviour }\end{array}$ \\
\hline \multicolumn{2}{|l|}{ Medical } \\
\hline - $\quad$ Nocturia & $\begin{array}{l}\text { Nocturnal pattern of behaviour that is } \\
\text { associated with incontinence or frequent } \\
\text { visits to the toilet }\end{array}$ \\
\hline - $\quad$ Pain & $\begin{array}{l}\text { Automatic recognition of pain through } \\
\text { facial expressions using machine learning } \\
\text { is in development [46]-[49]. }\end{array}$ \\
\hline - Constipation & $\begin{array}{l}\text { Recognition of the cyclic pattern of } \\
\text { challenging behaviour that is associated } \\
\text { with the cyclicity of bowel movements. }\end{array}$ \\
\hline \multicolumn{2}{|l|}{ Medications (ie, rule out drug-induced delirium) } \\
\hline $\begin{array}{l}\text { Anticholinergic drugs, Benzodiazepines, } \\
\text { Cholinesterase inhibitors, Digoxin, Opioids, } \\
\text { Substance abuse }\end{array}$ & $\begin{array}{l}\text { Detection of temporal relationship though } \\
\text { pattern recognition. }\end{array}$ \\
\hline
\end{tabular}

